distortion effects, it can equally well, in principle, be attributed to lattice strains alone. On the basis of certain assumptions, which will be described elsewhere, it has been calculated that, for lattice-distortion broadening, the ratio of the breadths from filings and from bulk metal strained in uniaxial tension should be of the order of $2-2 \cdot 5$ for corresponding $h k l$ reflexions.

Further analysis of the results in terms of lineshapes rather than line-breadths is now in progress.

We are indebted to the Chief Scientist, Department of Supply, Australia, for permission to publish this letter.

Defence Research Laboratories,

$$
\text { J. H. Auld }
$$

R. I. Garrol

Melbourne, Victoria,

Australia.

Oct. 31 .

${ }^{1}$ Wood, Proc. Roy. Soc., A, 172, 231 (1939).

${ }^{2}$ Brindley, Proc. Phys. Soc., 52, 117 (1940).

${ }^{3}$ Stokes, Pascoe and lipson, Nature, 151, 137 (1943).

' Smith and Stickley, Phys. Rev., 64, 191 (1943).

${ }^{5}$ Megaw and Stokes, J. Inst. Metals, 71, 279 (1945).

${ }^{6}$ Wood and Rachinger, J. Inst. Metals, 75, 571 (1949).

${ }^{2}$ Stokes and Wilson, Proc. Phys. Soc., 56, 174 (1944).

8 Stokes, Proc. Phys, Soc, B1, 382 (1948).

'Hall, Proc. Phys. Soc., 62, 741 (1949).

to Warren and Averbach, J. App. Phus., 21, 595 (1950).

\section{Screw Dislocations in Mica}

DIRECr evidence for the existence of dislocations has so far been confined to known examples of spiral growth : beryl ${ }^{1}$, an $n$-paraffin ${ }^{2}$, silicon carbide ${ }^{3,4}$ and cadmium iodide ${ }^{5}$. We have now experimental evidence for the existence of screw dislocations in mica (muscovite).

Mica cleavage faces have been intensively studied by $\mathrm{S}$. Tolansky, ${ }^{6,7}$, who proved the existence of steps on such surfaces by means of multiple-beam interferometry. These steps have heights which are, in general, whole multiples of $20 \mathrm{~A}$., in some cases ${ }^{8} 10 \mathrm{~A}$. On all the interferograms published so far, they run all over the surface. This is, in fact, nearly always so. We have now observed on a carefully cleaved sheet of mica a step ending in the middle of the face. The accompanying photograph (Fig. 1) is a reflexion Fizeau interferogram of this face $(\lambda=5470 \mathrm{~A}$.), the inset being an enlargement of the interesting part. From the fringe displacement the step-height was estimated to be $H= \pm 140 \mathrm{~A}$. We made sure that the step was not due to the optical flat. The reference flat could be arranged in such a way that the fringes

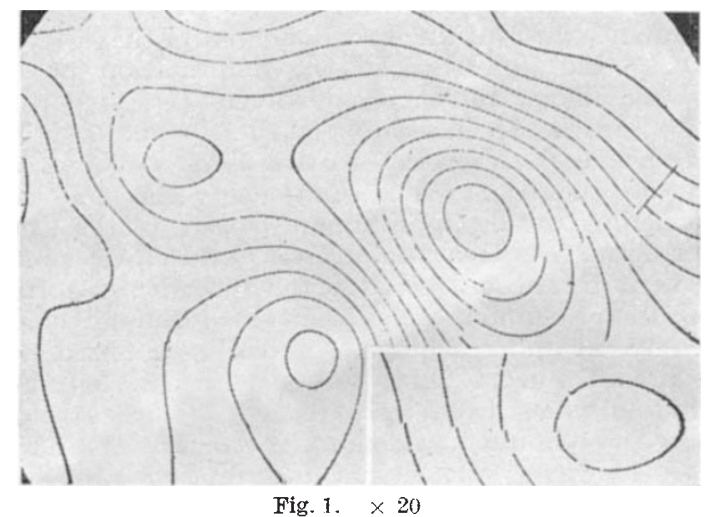

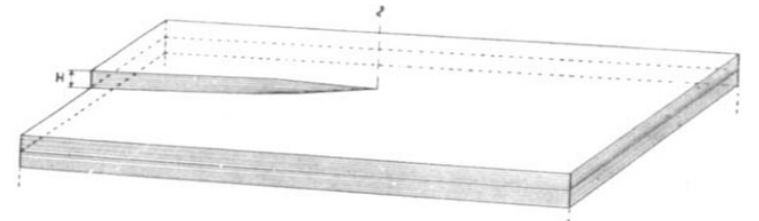

Fig. 2

surrounded the end-point of the step, so that we could conclude unambiguously that the step ended.

The surface topography corresponding to the fringe pattern is shown in Fig. 2. It is clear that the point where the step ends must be considered as the point where a screw dislocation emerges from the surface. As the step-height is equal to probably some seven times the identity distance, the screw dislocation has a resultant Burgers vector of some seven unit cells long. Dislocations having total strengths of the same order of magnitude are not exceptional, for example, in silicon carbide, as can be proved by measuring the step-heights of growth spirals on these crystals. Presumably, in this case also, the screw dislocation was generated during growth of the crystal and made it grow helicoidally, following the Frank mechanism ${ }^{9}$. As in mica the binding forces between sheets are much weaker than between ions in the sheet, it is to be expected that cleavage will proceed along the same plane (that is, the plane containing the potassium ions), even when passing a screw dislocation line. A coincidence of circumstances is, however, necessary to show the phenomenon: (1) a screw dislocation of suitable strength to produce a visible step must be present; (2) cleavage must take place without destroying the dislocation.

This explains why the phenomenon has hitherto escaped observation, and why a considerable number of pieces of mica had to be cleaved to obtain a photograph showing clear evidence.

The observations recorded here suggest a possible explanation for the various types of mica structures observed by S. B. Hendricks ${ }^{10}$, as well as for the chlorite structures. An account of interferometric observations on chlorites and sandwich structures of the chlorite type will be given elsewhere, in more detail, in collaboration with Prof. W. Dekeyser, to whom I am indebted for the remarks on the structure of mica and for suggesting this investigation. I also acknowledge the receipt of support from the C.N.B.C.P.M.

Note added in proof. Since this communication was submitted for publication, we have been able to observe growth spirals on biotite crystals"1 (var. meroxene, from Vesuvius) which give a direct proof of the preceding deductions.

Crystallographic Laboratory, Geological Institute, University of Ghent. Nov. 6.

${ }^{1}$ Griffin, L. J., Phil. Mag., 41, 196 (1950).

${ }^{2}$ Dawson, I. M., and Vand, V., Nature, 187, 476 (1951).

${ }^{3}$ Verma, A. R., Nature, 167, 939; 168, 431 (1951).

4 Amclinckx, S., Nature, 167, 939; 168, 431 (1951).

${ }^{5}$ Forty, A. J., Phil. Mag., 42, 670 (1951).

'Tolansky, S., Proc. Roy. Soc., A, 184, 51 (1945).

Tolansky, S., and Morris, P. G., Min. Mag., 198, 137 (1947).

${ }^{8}$ Courtney-Pratt, J. S., Research, 3, No. 1, 47 (1950).

- Frank, F. (., Farad. Soc. Discuss., No. 5, 48 (1949).

1" Hendricks, S. B., Amer. Min., 24, 729 (1939).

"Amelinckx, S., C.R. Acad. Sci., Paris, 234, 971 (1952). 